\section{BURST TRANSCRANIAL MAGNETIC STIMULATION FOR THE TREATMENT OF TINNITUS}

\section{To the Editor:}

We read with interest the report "Theta Burst Stimulation in the Treatment of Incapacitating Tinnitus Accompanied by Severe Depression," by Soekadar and colleagues. ${ }^{1}$ The authors describe the successful use of theta burst transcranial magnetic stimulation (TMS) of the temporoparietal cortex for the treatment of severe tinnitus. Here, we present a similar case and discuss available evidence for superiority of burst TMS in the treatment of tinnitus.

Single sessions of repetitive TMS (rTMS) over the temporal and temporoparietal cortex have been demonstrated to reduce tinnitus perception transiently. ${ }^{2,3}$ This effect has been observed both for high frequency rTMS $^{2,4}$ and for low-frequency rTMS. ${ }^{5,6}$ By investigating a large sample, de Ridder and colleagues $^{3}$ demonstrated that the optimal stimulation parameters for tinnitus suppression depend on clinical characteristics such as tinnitus duration. Perceptual characteristics of tinnitus also seem to play a role, since in patients with narrow band noise and white noise like tinnitus burst, TMS was superior to tonic TMS, whereas tonic and burst TMS were equally effective in patients with pure tone tinnitus. ${ }^{7.8} \mathrm{~A}$ recent study ${ }^{9}$ investigated systematically the effects of continuous, intermediate, or intermittent theta burst protocols. Approximately half of the patients reported a slight attenuation of tinnitus, but in the group analysis there was no significant difference between the different protocols. Taken together, the available data indicate that a large variety of different rTMS protocols interfere with tinnitus perception, but no specific protocol is clearly superior to others. This may also account for the effects of repeated sessions of rTMS, which are performed for the treatment of tinnitus. Repeated sessions of rTMS have been mainly performed with low frequency rTMS, since this intervention is assumed to reduce focal hyperactivity. ${ }^{10}$ However, a recent study has shown that high frequency rTMS with $10 \mathrm{~Hz}$ and $25 \mathrm{~Hz}$ are at least as effective as low frequency rTMS. ${ }^{11}$ This finding that clinical effects seem to be independent of rTMS frequency has resulted in the assumption that the therapeutic effect of rTMS might be mainly due to the interruption of tinnitus related neuronal activity in the auditory cortex. ${ }^{10,12}$ Nevertheless, the differential response to specific protocols in individual patients may reflect that there are different forms of tinnitus, which differ in their pathophysiology and, accordingly, in their response to specific therapeutic interventions.

For this reason we tested a variety of different rTMS protocols in a patient who had undergone previously 10 sessions of $1 \mathrm{~Hz}$ rTMS with limited success. After identifying the protocol which resulted in best transient tinnitus suppression, we applied this protocol in 10 further sessions and compared results with the standard treatment of $1 \mathrm{~Hz}$ rTMS

The 61-year-old man presented with unilateral high-pitched narrow band tinnitus on the right side, which had started 2 years before. Tinnitus severity was moderate with a score of 24 in the tinnitus questionnaire, ${ }^{13}$ tinnitus loudness was rated as 6 on a numeric rating scale ranging from $0-10$. Microscopic examination of the ear was normal. Pure tone audiometry was normal on the left side $(250 \mathrm{~Hz}-8 \mathrm{kHz}:<20 \mathrm{~dB} \mathrm{HL})$, on the right side there was a sloping audiogram with normal hearing until $1 \mathrm{kHz}$ and $30 \mathrm{~dB} \mathrm{HL}$ at $8 \mathrm{kHz}$. Pitch matching revealed a tinnitus frequency around $8 \mathrm{kHz}$.

Treatment with noise generators resulted in some relief during the day. However, the patient complained of disturbing tinnitusrelated insomnia. A fluorodeoxyglucose (FDG) positron emission topography scan demonstrated increased FDG uptake in the left auditory cortex (Figure 1). Ten sessions of neuronavigated rTMS over this area $(1 \mathrm{~Hz}$,

\section{FIGURE 1. FDG PET had been per- formed before treatment}

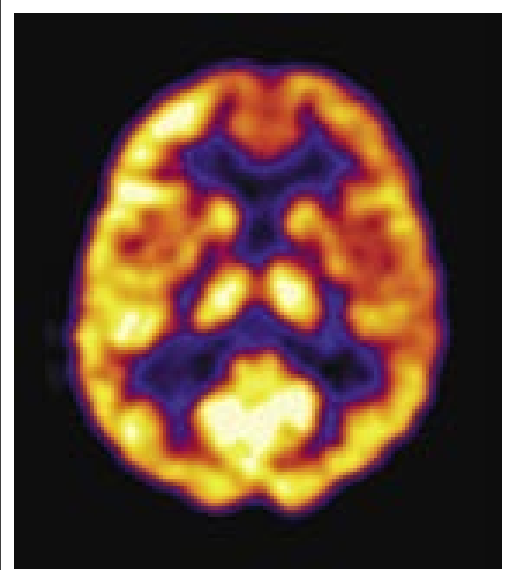

The area of hypermetabolic activity in the tempora cortex was chosen as a target for TMS treatment The transversal slice through the temporal brain region shows unilaterally increased metabolic activity in projection to the left auditory cortex.

$\mathrm{FDG}=\left[{ }^{18} \mathrm{~F}\right]$ deoxyglucose; $\mathrm{PET}=$ positron emission topography; TMS=transcranial magnetic stimulation.

Frank E, Landgrebe $M$, Kleinjung T, Hajak G, Langguth B. CNS Spectr. Vol 15, No 8. 2010.
$110 \%$ MT, 2000 stimuli; Medtronic MagPro Option) resulted in reduction of the patient's tinnitus severity, as reflected by the tinnitus questionnaire (TO) of Goebel and Hiller and the subjective clinical global impression score (Figure 2). However, during the following weeks tinnitus increased again and returned to baseline level 11 weeks after the end of rTMS treatment. At that time, different rTMS protocols were tested for immediate effects on tinnitus $(5 \mathrm{~Hz}$ tonic; $10 \mathrm{~Hz}$ tonic; $20 \mathrm{~Hz}$ tonic each 100 stimuli; $5 \mathrm{~Hz}$ burst, 100 bursts consisting of 3 stimuli with a frequency of $50 \mathrm{~Hz}$ [continuous theta burst]; 10 $\mathrm{Hz}$ burst, 100 bursts consisting of 3 stimuli with a frequency of $100 \mathrm{~Hz} ; 20 \mathrm{~Hz}$ burst, 100 bursts consisting of 5 stimuli with a frequency of $500 \mathrm{~Hz}$ ). Treatment with $20 \mathrm{~Hz}$ burst TMS resulted in transient tinnitus reduction of $65 \%$, whereas sham rTMS did not influence tinnitus. Twenty $\mathrm{Hz}$ burst TMS was then applied for 2 weeks with five treatment sessions per week (2,000 bursts/day; 40 trains with 50 bursts and an intertrain interval of 25 seconds).

After 10 days of treatment the effect of the burst stimulation protocol was of similar magnitude to the standard treatment with 1 $\mathrm{Hz}$. However, the effect was more stable over the follow-up period (Figure 2). Improvement on the TO between days 90 and 100 may reflect an anticipation effect, since at day 90 the test stimulation with different stimulation protocols was performed and the decision was made to perform a second rTMS treatment series.

\section{FIGURE 2. \\ Change of tinnitus after 10 days of $1 \mathrm{~Hz}$ tonic rTMS to the left temporal cortex and after 10 days of $20 \mathrm{~Hz}$ burst rTMS to the left temporal cortex}

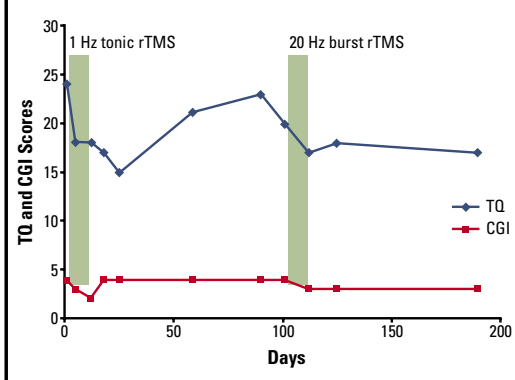

rTMS=repetitive transcranial magnetic stimula tion; TQ=Tinnitus Questionnaire; CGI=Clinical Global Impression-Change.

CGI: $4=$ no change; $3=$ slightly better; $2=$ much better.

Frank $E$, Landgrebe $M$, Kleinjung $T$, Hajak $G$, Langguth B. CNS Spectr. Vol 15, No 8. 2010. 
This case of a patient who experienced similar treatment effects of a standard protocol of $1 \mathrm{~Hz}$ tonic rTMS and an individualized protocol of $20 \mathrm{~Hz}$ burst rTMS further supports the notion that rTMS treatment effects do not primarily depend on the stimulation frequency. The longer-lasting treatment effects after the second treatment series may suggest superiority of the $20 \mathrm{~Hz}$ burst protocol, which was chosen since it was most effective for transient tinnitus reduction. An alternative explanation for the longer lasting treatment effects of the second treatment may be that repeated sessions of rTMS result in longer-lasting treatment effects independently from the stimulation frequency ${ }^{14,15}$ Hence, the observed reduction of tinnitus after continuous theta burst TMS in the case presented by Soekadar and colleagues ${ }^{1}$ might also have occurred with another stimulation frequency.

Head-to-head comparison studies with large sample sizes will enable us to answer the question of whether specific rTMS treatment protocols are superior to others in specific patient subgroups and whether individual testing for the most efficient stimulation frequency can increase treatment efficacy. A further approach is the assessment of the effects of the different protocols on neuronal function by electrophysiologic or imaging methods.

\section{Sincerely,}

Elmar Frank, MD

Michael Landgrebe, MD

Tobias Kleinjung, MD

Göran Hajak, MD

Berthold Langguth, MD

\section{References}

. Soekadar SR, Arfeller C, Rilk A, Plontke SK, Plewnia C. Theta burst stimulation in the treatment of incapacitating tinnitus accompanied by severe depression. CNS Spectr. 2009;14(4):208-211.

2. Plewnia C, Bartels M, Gerloff C. Transient suppression of tinnitus by transcranial magnetic stimulation. Ann Neurol 2003;53:263-266

3. De Ridder D, Verstraeten E, Van der KK, et al. Transcrania magnetic stimulation for tinnitus: influence of tinnitus duration on stimulation parameter choice and maximal tinnitus suppression Otol Neurotol. 2005-26(4):616-619.

4. Folmer RL, Carroll JR, Rahim A, Shi Y, Hal MW. Effects of repetitive transcranial magnetic stimulation (rTMS) on chronic tinnitus. Acta Otolaryngol Suppl. 2006;(556):96-101

5. Londero A, Lefaucheur JP, Malinvaud D, et al. [Magnetic stimulation of the auditory cortex for disabling tinnitus: preliminary results]. Presse Med 2006:35(2 Pt 1):200-206.

6. Plewnia C, Reimold M, Najib A, et al. Dose-dependent attenuation of auditory phantom perception (tinnitus) by PET-guided repetitive transcranial magnetic stimulation. Hum Brain Mapp. 2007;28(3):238-246

7. De Ridder D, van der Loo E, Van der Kelen K Menovsky T, Van de Heyning P. Moller A. Do tonic and burst TMS modulate the lemniscal and extralemniscal system differentially? Int J Med Sci. 2007:4(5):242-246

8. De Ridder D, van der Loo E, Van der Kelen K, Menovsky T, Van de Heyning P, Moller A. Theta, alpha and beta burst transcranial magnetic stimulation: brain modulation in tinnitus. Int $J$ Med Sci. 2007:4(5):237-241.

9. Poreisz C, Paulus W, Moser T, Lang N. Does a single session of theta-burst transcranial magnetic stimulation of infe- rior temporal cortex affect tinnitus perception? BMC Neurosci. 2009:10:54

10. Langguth B, De Ridder D, Dornhoffer JL, et al. Does rTMS/tDCS show efficacy in treating tinnitus patients? Brain Stimulation. 2008:1(3):192-205

11. Khedr EM, Rothwell JC, Ahmed MA, El Atar A. Effect of daily repetitive transcranial magnetic stimulation for treatment of tinnitus: comparison of different stimulus frequencies. $J$ Neurol Neurosurg Psychiatry. In press.

12. Khedr EM, Rothwell JC, Ahmed MA Awad EM, Galal 0 Cortical excitability and transcallosal inhibition in chronic tinnitus: transcranial magnetic study. Neurophysiol Clin. 2008:38(4):243-248.

13. Goebel G, Hiller W. The tinnitus questionnaire. A standard instrument for grading the degree of tinnitus. Results of a multicenter study with the tinnitus questionnaire. Hno. 1994;42:166-172

14. Mennemeier M, Chelette KC, Myhill J, et al. Maintenance Repetitive Transcranial Magnetic Stimulation Can Inhibit the Return of Tinnitus. Laryngoscope. 2008;118(12):1228-1232

15. Langguth B, Landgrebe M, Hajak G, Kleinjung T. Maintenance repetitive transcranial magnetic stimulation can inhibit the return of tinnitus. Laryngoscope 2008:118(12)'2264-2265.

Drs. Frank and Landgrebe are senior psychiatrists, and Dr. Hajak is vice director in the Departmen of Psychiatry at the University of Regensburg in Germany. Dr. Langguth is senior psychiatrist in the Department of Psychiatry and head of the Tinnitus Center, both at the University of Regensburg. Dr. Kleinjung is senior otolaryngeologist in the Department Kleinjung is senior otolaryngeologist in the Department of Otorhinolaryngology and head of the
Center, both at the University of Regensburg.

Faculty Disclosures: The authors report no affiliation with or financial interest in any organization that might pose a conflict of interest.

Submitted for publication: May 8, 2009; Accepted for publication: December 1, 2009.

Please direct all correspondence to: Berthold Langguth, MD, Department of Psychiatry, University of Regensburg, Universitaetsstrasse 84, D-93053 Regensburg, Germany. Tel: +49-941-941-2099 Regensburg, Germany. Tel: +49-941-941-2099,
Fax: +49-941-941-2025; E-mail: Berthold. Langguth@medbo.de.

\section{Response from the Author:}

We thank Dr. Frank and colleagues for their comment on our case ${ }^{1}$ and their own interesting case report. We agree with the authors' conclusion that repetitive transcranial magnetic stimulation (rTMS) treatment effects are not primarily depending on the stimulation frequency, which is consistent with our findings implying that in addition to low- and high-frequency rTMS, theta burst stimulation might also have an ameliorating effect on tinnitus. However, we would like to comment on some methodological and neurophysiological aspects concerning the case report and its discussion.

First, transient modulation of tinnitus loudness has been reliably demonstrated by means of different rTMS paradigms inducing short-term interference with, ${ }^{2-4}$ or attenuation of ${ }^{5}$ cortical activity. These findings have proven the functional relevance of temporoparietal cortical areas for the pathophysiology of tinnitus. However, the effectiveness and clinical relevance of repeated sessions of rTMS targeting this area is still under debate and lacks robust data. ${ }^{6}$ Furthermore, the mechanism underlying this intended therapeutic effect is not clear. The therapeutic value, however, has to be demonstrated in larger randomized controlled trials, preferably on the basis of a well-defined pathophysiological model., ${ }^{7,8}$ Frank and colleagues have observed a significant immediate effect ( $68 \%$ reduction) of $20 \mathrm{~Hz}$ burst stimulation. Based on this finding, they decided to treat their patient using these parameters. However, the predictive value of immediate transient effects is unknown. Most likely, these "virtual lesion effects" are based on interference with ongoing cortical activity. In contrast, the long-term therapeutic efficacy is presumably mediated by fundamentally different processes. Accordingly, the therapeutic outcome is much smaller than the reported immediate reduction of tinnitus.

Second, low-frequency rTMS and theta burst stimulation both aim for a reduction of cortical hyperactivity associated with tinnitus perception. Based on this mechanistic model of rTMS action, various frequencies resulting in a decrease of cortical excitability are suitable to reduce tinnitus perception. However, it is important to account for the specific stimulation parameters used in the treatment of the patient reported here. In contrast to the well-known $1 \mathrm{~Hz}$ rTMS and continuous theta burst stimulation, in this case $500 \mathrm{~Hz}$ rTMS applied at a burst frequency of $20 \mathrm{~Hz}$ was used. The neurophysiological implications of this kind of stimulation are yet unknown. Therefore, conclusions about mechanism in respect of tinnitus reduction cannot be drawn. Although it is interesting that these stimulation parameters reduce tinnitus distress by $\sim 15 \%$ (3 points of the Tinnitus Questionnaire [TQ]), the potential therapeutic effect of other stimulation parameters are unknown. It appears that the numerous possible combinations of stimulation parameters require a rational approach based on well-defined pathophysiological models.

Finally, the authors attribute the tinnitus improvement of 3 points TQ prior to the start of the $20 \mathrm{~Hz}$ treatment (days 90 to 100) to an "anticipation effect." If so, this nonspecific influence has to be also considered a cause for the reduction of further 3 points after 5 days of $20 \mathrm{~Hz}$ treatment, pointing toward a placebo-like effect.

In summary, we agree with the authors' conclusion that the observed reduction of tinnitus after continuous theta burst stimulation presented in our case ${ }^{1}$ might also have occurred at another stimulation frequency. However, we do not agree that Frank and colleagues present evidence for the notion that particularly $20 \mathrm{~Hz}$ burst treatment was superior to $1 \mathrm{~Hz}$ rTMS in the reported case and that individual pre-screening of stimulation frequencies provides relevant information on subsequent treatment response. The effect size of $1 \mathrm{~Hz}$ rTMS and $20 \mathrm{~Hz}$ burst 
TMS treatment and the difference of therapeutic efficacy between these stimulation protocols reported in this case are small and in the range of unspecific "anticipation effects" or common fluctuations of tinnitus distress. However, this case supports the view that the mechanisms and effects of immediate and long-term effects are most likely different. It also illustrates that the neurophysiological features of a stimulation protocol should be reckoned as an essential factor for developing new therapeutic approaches using brain stimulation for the exploration and treatment of chronic tinnitus.

Sincerely,

Christian Plewnia, MD

\section{References}

Soekadar SR, Arfeller C, Rilk A, Plontke SK, Plewnia C. Theta burst stimulation in the treatment of incapacitating tinnitus accompanied by severe depression. CNS Spectr. 2009;14:208-211.

2. Plewnia $\mathrm{C}$, Bartels $\mathrm{M}$, Gerloff $\mathrm{C}$. Transient suppression of tinnitus by transcranial magnetic stimulation. Ann Neurol. 2003:53:263-266

3. De Ridder D, Verstraeten E, Van der KK, et al. Transcrania magnetic stimulation for tinnitus: influence of tinnitus duration on stimulation parameter choice and maximal tinnitus suppression. Otol Neurotol. 2005;26:616-619.

4. Fregni F, Marcondes R, Boggio PS, et al. Transient tinnitus suppression induced by repetitive transcranial magnetic stimulation and transcranial direct current stimulation. Eur J Neurol. 2006;13:996-1001

5. Plewnia C, Reimold M, Najib A, et al. Dose-dependent attenuation of auditory phantom perception (tinnitus) by PET-guided repetitive transcranial magnetic stimulation. Hum Brain Mapp. 2007:28:238-246.

6. Plewnia C. Brain stimulation: New vistas for the exploration and treatment of tinnitus. CNS Neurosci Ther. 2010. In press.

7. Landgrebe $\mathrm{M}$, Binder $\mathrm{H}$, Koller $\mathrm{M}$, et al. Design of a placebo-controlled, randomized study of the efficacy of repetitive transcranial magnetic stimulation for the treatment of chronic tinnitus. BMC Psychiatry 2008:8.23.

8. Arfeller C, Vonthein R, Plontke SK, Plewnia C. Efficacy and safety of bilateral continuous theta burst stimulation (cTBS) for the treatment of chronic tinnitus: design of a three-armed randomized controlled trial. Trials. 2009;10:74.

Dr. Plewnia is senior psychiatrist and head of the Brain Stimulation Laboratory in the Department of Psychiatry and Psychotherapy at the University of Tuebingen in Germany.

\section{LAMOTRIGINE-ASSOCIATED DEPERSONALIZATION SYMPTOMS}

\section{To the Editor:}

Lamotrigine has been approved by the Food and Drug Administration for the treatment of epilepsy and for the maintenance phase of bipolar disorder. The most common adverse events described with the use of lamotrigine are severe skin reactions, insomnia, headache, dizziness, and ataxia.

In the prescribing information presented to the FDA, depersonalization symptoms are an infrequent form of adverse reaction. ${ }^{1}$
According to lamotrigine prescribing information, the precise mechanism of action is unknown. In vitro studies suggest that it stabilizes neuronal membranes by blocking sodium channels and consequently modulating presynaptic transmitter release of excitatory amino acids like Glutamate. ${ }^{1}$ Some data on in vitro and in vivo research may support the hypothesis of lamotrigine glutamate modulation. ${ }^{2,3}$

It has been shown that depersonalization-related phenomena may be produced by glutamate hyperactivity, and there are reports that lamotrigine can ameliorate these symptoms. ${ }^{4,5}$ In accordance with this hypothesis, we describe paradoxical depersonalization symptoms that were likely induced by lamotrigine.

A 54-year-old man consulted his doctor due to the recurrence of depression symptoms in a context of a bipolar II disorder. He was started on quetiapine $25 \mathrm{mg} /$ day and gradually titrated to $150 \mathrm{mg} /$ day. After 1 month of treatment the patient remained with residual depressive symptoms. Lamotrigine $25 \mathrm{mg} /$ day was added and titrated to 200 $\mathrm{mg} /$ day in 1 month. With this pharmacologic treatment the patient achieved euthymia.

Fifteen days after the $200 \mathrm{mg} / \mathrm{day}$ lamotrigine regimen, the patient described a suddenly perception of "out of body experience" with a length of no more than 5 seconds. He then alternated symptoms of perception of being much taller than usual, changes in his voice tone, and the perception of "sinking" on the floor while he was walking. According to the patient, these symptoms appeared no more than one or two times a day, and lasted 5-10 seconds, within a context of clear sensorium.

Due to patient complaints, the dosage of lamotrigine was gradually reduced. After lamotrigine reduction the frequency and intensity of depersonalization decreased, and disappeared with lamotrigine suspension. The patient continued with a pharmacologic regimen of quetiapine $150 \mathrm{mg} /$ day and valproate $250 \mathrm{mg} /$ day without depersonalization symptoms.

After checking patient medical records, it was found that the patient had taken lamotrigine 7 years ago for almost 1 month. He started with $12.5 \mathrm{mg} /$ day and was titrated up $75 \mathrm{mg} /$ day. The patient did not remember this prescription, but the cause of lamotrigine suspension was due to the emergence of ataxia and depersonalization symptoms. ${ }^{4}$

In addition to lamotrigine-induced depersonalization symptoms, other explanations for the symptoms in this case have been considered.

One explanation for the patient's symptoms could be the natural emergence of depersonalization symptoms during an acute depressive state. This patient had many depressive phases during the course of his illness, but depersonalization symptoms were only present with the addition of lamotrigine.

An alternative cause to consider is that depersonalization symptoms may be related to quetiapine. ${ }^{6}$ However, depersonalization symptoms disappeared after lamotrigine suspension despite the continuation of quetiapine. Thus, it is unlikely that quetiapine was a primary cause of this symptom.

Lamotrigine and quetiapine serum levels haven not been searched, but the patient had a history of a good compliance and adherence in his $>15$ years of treatment.

It should also be noted that due to the patient's clinical state, lamotrigine was titrated very quickly in comparison to the recommendations in the medication's prescribing information. ${ }^{1}$ This fact may have precipitated the adverse reaction, but the patient also had these symptoms with $75 \mathrm{mg} /$ day titrated in a month.

This case illustrates an infrequent case of depersonalization symptoms that, according to the Naranjo adverse drug reaction estimating scale, is a probable adverse event triggered by lamotrigine (7 points: 01 , $0 ; 02,2 ; 03,1 ; 04,2 ; 05,-1 ; 06,1 ; 07,0 ; 08,1$; $09,1 ; 010,0){ }^{7}$

Sincerely,

Francisco Appiani, MD

Brendan T. Carroll, MD

\section{References}

1. Available at: www.accessdata.fda.gov/drugsatfda_docs/ label/2009/020241s037s038,020764s030s031 lbl.pdf. Accessed August 26, 2009

2. Wang SJ, Sihra TS, Gea PW. Lamotrigine inhibition of glutamate release from isolated cerebrocortical nerve terminals (synaptosomes) by suppression of voltage-activated calcium channe activity. Neuropharmacol Neurotoxicol. 2001;12(10):2255-2258. 3. Anand A, Charney DS, Oren D, et al. Attenuation of neuropsychiatric effects of ketamine with lamotrigine. Arch Gen Psychiatry. 2000;57:270-276

4. Sierra M, Phillips M, Ivin G, et al. A placebo-controlled, cross-over trial of Lamotrigine in depersonalization disorder. $J$ Psychopharmacol. 2003:17(1):103-105.

5. Medford N, Sierra M, Baker D, David A. Understanding and treating depersonalization disorder. Advances in Psychiatric Treatment. 2005;11:92-100.

6. Sarkar J, Jones N, Sullivan G. A case of depersonalizationderealization syndrome during treatment with Quetiapine. $J$ Psychopharmacol 2001:15(3):209-211.

7. Naranjo CA, Busto U, Sellers EM, et al. A method for estimating the probability of adverse drug reactions. Clin Pharmacol Ther. 1981;30:239-245

Dr. Appiani is assistant professor of pharmacology at the University of Buenos Aires, and director of aCEDEN in Buenos Aires, Argentina. Dr. Carroll is clinical assistant professor of psychiatry at Ohio University College of Osteopathic Medicine, and chief of Psychiatry Service at the VA Medical Center in Chillicothe, Ohio.

Faculty Disclosures: Dr. Appiani reports no affiliation with or financial interest in any organization that migh pose a conflict of interest. Dr. Carroll is an unpaid consultant to the Neuroleptic Malignant Syndrome consultant to the Neuroleptic Malignant Syndrome Information Service; and is on the speaker's bureaus
of Abbott, AstraZeneca, Bristol-Myers Squibb, Eli Lilly, 
Forest Laboratories, Janssen, and Pfizer.

Submitted for publication: May 15, 2009; Accepted for publication: December 7, 2009.

Please direct all correspondence to: Francisco Appian MD, ACEDEN (Asociación Civil para el Estudio Desarrollo de las Neurociencias), Arenales 2838 5 B (CP: 1425), Buenos Aires, Argentina. Tel/Fax: 54-114-822-7360; E-mail: Franciscoappiani@live. com.ar.

\section{AUDITORY HALLUCINATIONS ASSOCIATED WITH HEAD- ACHES FOLLOWING TRAUMATIC BRAIN INJURY}

\section{To the Editor:}

Traumatic brain injury (TBI) can lead to significant neurological damage, and is strongly associated with chronic headaches, ${ }^{1}$ seizure disorder, ${ }^{2}$ cognitive impairment, ${ }^{3}$ sleep disorders, ${ }^{4}$ and psychiatric disorders. ${ }^{5}$ The prevalence of such impairments in TBI patients appears to be quite high, with $>20 \%$ of patients experiencing at least some neurocognitive deficits that can persist for decades. ${ }^{3,5,6}$ Over 1 million cases of TBI occur every year and often disable otherwise young and healthy patients, making a significant impact on healthcare as a whole.

The prevalence of certain psychiatric disorders following TBI has been extensively studied, and it is now known that head trauma is associated with substantial increases in depression, ${ }^{3,7-11}$ anxiety, ${ }^{7}$ ${ }^{10}$ and personality disorders. ${ }^{7-9,12}$ Psychosis due to TBI (PDTBI) is another less common psychiatric diagnosis made in patients who exhibit hallucinations or delusions following a significant head injury. ${ }^{7,13}$ This is a difficult diagnosis to make, since the clinician must decide whether the psychosis can be better explained by othe psychiatric disorders that may have incidentally occurred in spite of the injury.

Chronic headaches are another common and sometimes disabling complication of TBI, occurring in $>30 \%$ of patients. ${ }^{14}$ Headaches, especially migraines, have been reported to associate with visual, olfactory, gustatory, and auditory hallucinations. ${ }^{15}$ Most auditory hallucinations associated with headaches consist of relatively simple perceptions such as tinnitus or hearing loss, ${ }^{16}$ while complex hallucinations with discernable voices or other sounds are very rare.

In this report, we present the case of a young male who experienced significant head trauma, and then immediately developed intense daily headaches. Several years later, he developed command audi- tory hallucinations that occurred exclusively during these headaches.

The patient is a 40 -year-old Asian male who was admitted voluntarily to an inpatient psychiatric ward in the Southwest. He complained of hearing command auditory hallucinations during a headache that told him to "get better" by taking an entire bottle of medication. The patient subsequently ingested a bottle of olanzapine tablets, and then called 911 for help.

In 1990 at 21 years of age, the patient was healthy with no medical or psychiatric problems, and no family history of psychiatric disease. He was working in a factory and was struck on the left parietotemporal region of the head by a heavy metal bar, which resulted in a skull fracture and loss of consciousness for $\sim 30$ minutes. After the accident, the patient began to have severe headaches several times a week that were described as steady bitemporal pain lasting between 30 minutes to 2 hours. The headaches were worse when he had not slept well the night before, which he reports being a particular problem since he also had frequent episodes of primary insomnia following the head injury. He found that sleeping well and taking painkillers usually offers some relief.

Immediately after the accident, the patient began to show some personality changes, burning clothes in his backyard and worrying that he was possessed by a ghost. He also became increasingly suspicious, believing that people at his work were plotting against him. Four years after the initial injury, the patient developed auditory hallucinations which would occur exclusively when he was having a headache. He described the auditory hallucinations as male and female voices that speak to him and give him commands, typically instructing him to do menial tasks such as turning his television on and off repeatedly. At times, however, the voices become more prominent and will instruct him to overdose on medication or break windows so that he can "escape" from his home. These hallucinations occur during almost every headache, and can occur at any time during it.

In 1995, the patient was admitted for the first time to a psychiatric facility for medication overdose secondary to instructions from his command hallucinations. In the following years he had several more psychiatric admissions, each time overdosing on medications such as acetaminophen and antipsychotics after hearing command voices telling him to do so. During each admission, the patient always denied suicidal intent, but felt compelled to obey the commands.

On the present admission, physical examination showed a well-developed Asian male with no superficial evidence of head trauma and no focal neurological deficits (including during headaches). He had a blunted affect but showed no signs of paranoia or active hallucinations, and showed considerable insight into his condition, admitting that the voices were not real and that he needed help for this problem.

The patient showed intact recent and remote memory, with normal concentration. Laboratory analysis showed normal electrolytes, liver function tests, thyroid function, and normal urinalysis with no drugs of abuse. Computed tomography of the head from a prior admission several years prior showed no residual brain or skull injuries; the original skull $x$-ray films from the accident were not available.

As an outpatient, the patient had been taking olanzapine $10 \mathrm{mg} / \mathrm{day}$. On admission to the inpatient unit, the patient was continued on this, and given trazodone for sleep and a combination of acetaminophen and ibuprofen for headaches. Within 2 days the patient's sleep improved from 4 hours to 6 hours at night, and he reported less intense headaches with significant reduction in hallucinations. He was discharged home on olanzapine $20 \mathrm{mg} /$ day to his family after a 3-day stay.

The patient presented in this report fits the typical profile of a patient suffering from PDTBI. Most patients with PDTBI reported in the literature ${ }^{17}$ fit a similar profile to this patient in that they are male, relatively young at the time of injury, suffer a severe head injury with loss of consciousness, have a several year latency to the first signs of psychosis, and typically present with auditory hallucinations as a positive symptom of psychosis. The unusual characteristic of this case is the strong association between the headaches and the command auditory hallucinations that later followed.

Since the link between head injury and later psychosis is often vague and circumstantial at this point, it will be of interest to determine whether other cases of likely PDTBI similarly have psychotic symptoms related to other neurological complaints that have clearly followed the initial head injury. If such associations can be found in larger studies, it may establish addi- 
tional future criteria to help make this difficult diagnosis more certain.

\section{Sincerely,}

Jadon Webb, MD, PhD

John Quinn, MD

Arthur Westover, MD

\section{References}

1. Faux S, Sheedy J. A prospective controlled study in the prevalence of posttraumatic headache following mild traumatic brain injury. Pain Med. 2008;9(8):1001-1011.

2. Ferguson PL, Smith GM, Wannamaker BB, et al. A populationFerguson PL, Smith GM, Wannamaker BB, et al. A population-
based study of risk of epilepsy after hospitalization for traumatic brain injury. Epilepsia. 2009;51(5):891-898.

3. Hoofien D, Gilboa A, Vakil E, Donovick PJ. Traumatic brain injur (TBI) 10-20 years later: a comprehensive outcome study of psychiatric symptomatology, cognitive abilities and psychosocial functioning. Brain Inj 2001:15(3):189-209.

4. Orff HJ, Ayalon L, Drummond SP. Traumatic brain injury and sleep disturbance: a review of current research. $J$ Head Trauma Rehabil. 2009;24(3):155-165.

5. Vaishnavi S, Rao V, Fann JR. Neuropsychiatric problem after traumatic brain injury: unraveling the silent epidemic. Psychosomatics, 2009;50(3):198-205

6. von Wild KR. Posttraumatic rehabilitation and one year outcome following acute traumatic brain injury (TBI): data from the well defined population based German Prospective Study 2000 2002. Acta Neurochir Suppl. 2008;101:55-60.

7. Whelan-Goodinson R, Ponsford J, Johnston L, Grant F.Psychiatric disorders following traumatic brain injury: their nature and frequency. J Head Trauma Rehabil. 2009;24(5):324-332.

8. Koponen S, Taiminen T, Portin R et al. Axis I and II psychiatric disorders after traumatic brain injury: a 30 -year follow-up study. Am J Psychiatry. 2002;159(8):1315-1321.

9. Koponen S, Taiminen T, Kurki T, et al. MRI findings and Axis I and II psychiatric disorders after traumatic brain injury: a 30-year retrospective follow-up study. Psychiatry Res. 2006;146(3):263-270.

10. Fann JR, Katon WJ, Uomoto JM, Esselman PC. Psychiatric disorders and functional disability in outpatients with traumatic brain injuries. Am J Psychiatry. 1995;152(10):1493-1499.

11. Jorge RE, Robinson RG, Moser D, et al. Major depression following traumatic brain injury. Arch Gen Psychiatry 2004;61(1):42-50

12. Hibbard MR, Bogdany J, Uysal S, et al. Axis II psychopathology in individuals with traumatic brain injury. Brain $I n j$. 2000:14(1):45-61.

13. Harrison G, Whitley E, Rasmussen F, et al. Risk of schizophrenia and other non-affective psychosis among individuals exposed to head injury: case control study. Schizophr Res. 2006;88(1
3):119-126.

14. McAbee G, Feldman-Winter L. Auditory hallucinations in migraine without psychosis. J Child Neurol. 1999:14(7):479-80. 15. Morrison DP. Abnormal perceptual experiences in migraine. Cephalalgia. 1990;10(6):273-277.

16. Rubin D, McAbee GN, Feldman-Winter LB. Auditory hallucinations associated with migraine. Headache. 2002;42(7):646-648. 17. Fujii D, Ahmed I. Characteristics of psychotic disorder due to traumatic brain injury: an analysis of case studies in the literature. J Neuropsychiatry Clin Neurosci. 2002;14(2):130-140.

Dr. Webb is medical resident, Dr. Quinn is associate professor, and Dr. Westover is assistant professor in the Department of Psychiatry at the University of Texas Southwestern Medical Center in Dallas.

Faculty Disclosures: The authors report no affiliation with or financial interest in any organization that may pose a conflict of interest.

Submitted for publication: January 25, 2010; Accepted for publication: June 4, 2010.

Please direct all correspondence to: Jadon Webb, $M D, P h D$, Department of Psychiatry, University of Texas Southwestern Medical Center, 5323 Harry Hines Blvd, Dallas, TX 75390; Tel: 214-629-9322; E-mail: Jadon_Webb@yahoo.com. 